\title{
Elastic and mechanical properties of cubic metal arsenides (Ga, In and Al) under high-pressure: a simulation study
}

\author{
Nenuwe Oyindenyifa Nelson* and Umukoro Judith \\ Department of Physics, Federal University of Petroleum Resources, PMB 1221, Effurun, Delta State, \\ Nigeria \\ *Correspondence: nenuwe.nelson@fupre.edu.ng; (D) ORCID: https://orcid.org/0000-0002-3112-3869
}

Received: $26^{\text {th }}$ March 2020, Revised: $6^{\text {th }}$ June 2021, Accepted: $19^{\text {th }}$ June 2021

\begin{abstract}
Semiconducting materials have played an important role in modern technological age. Group III-V materials have attracted much attention in electronic industry due to their structural, mechanical, electronic and thermodynamic properties predicted by calculations. This paper simulated the effect of pressure within the range of 0-100 GPa on the elastic constants and other related parameters, such as Young's, bulk and shear moduli, Pugh ratio, Poisson ratio, anisotropy factor, degree of anisotropy and Kleinman parameter for gallium arsenide (GaAs), indium arsenide (InAs) and aluminum arsenide (AlAs) materials, using the Tersoff classical potential within ATK-force field. Results showed that, increase in pressure enhanced the ductility of GaAs and InAs within the entire pressure domain, and between 10-40 GPa for AlAs material. AlAs was found to be brittle under 50-90 GPa, and unstable at 100 $\mathrm{GPa}$. This may be due to occurrence of phase transition at these pressures. The obtained results at zero pressure are consistent with available experimental and theoretical data in literature.
\end{abstract}

Keywords: Elastic constants, ductility, gallium arsenide, high pressure, indium arsenide.

\section{Introduction}

The knowledge of high-pressure dependence of the elastic parameters is critical for predicting some physics of semiconducting materials. For instance, high-pressure analysis gives vital information about the phase transition, stability, strength, elastic, and mechanical properties of semiconductors (Liu et al. 2007, Zhu et al. 2008, Liu et al. 2009, Wang et al. 2009, Guler and Guler 2014). Currently, III-V compound semiconductors, such as gallium arsenide (GaAs), indium arsenide (InAs) and aluminum arsenide (AlAs) are the major materials for micro/nano/opto-electronic 
device applications. They exhibit excellent optical, elastic, mechanical, and electronic properties which make them suitable for applications and have received considerable interest from experimental and theoretical researchers. GaAs is used in photovoltaics, semiconductor lasers, light emitting diodes, solar cells and heterostructures (Blakemore 1982, Rong et al. 2008, Al-Douri and Ali 2011). AlAs is used in high electric mobility transistors, solid state lasers, Bragg reflector super lattices and heterojunction bipolar transistors (Liu 1995, Li et al. 2013), and InAs is used in infrared detectors, photodiodes, and tetrahertz radiation source (Lide 1998).

Elastic parameters provide connection between mechanical and dynamic property of cubic crystals and give insight into the nature of forces operating in materials. Under standard conditions, GaAs, InAs and AlAs crystallize in cubic zinc-blend structure. At high pressure, these materials are found to undergo structural phase transition, thereby exhibiting different mechanical properties. Phase transition and elasticity of GaAs was reported by Guller and Guller (2014) up to $25 \mathrm{GPa}$ from Geometry Optimization technique. Li et al. (2013) reported the pressure dependence of elastic and lattice dynamics properties of AlAs from ab initio studies up to $15 \mathrm{GPa}$. Also, Kabita et al. (2016) studied structural, elastic, and electronic properties of InAs under induced pressure within the range of 0-4 GPa, and Louail et al. (2006) reported the elastic properties of InAs under pressure up to $18 \mathrm{GPa}$, using the CASTEP density functional theory.

Although, there have been studies on elastic and mechanical properties of these binary compounds under ambient conditions, experimental and theoretical investigations of these properties under high pressure are still scanty in literature. Therefore, the objective of this simulation work is to reveal the behaviour of elastic and mechanical properties of GaAs, InAs and AlAs under pressure in the range of 0 $100 \mathrm{GPa}$.

\section{Computational Methods}

The computation of elastic parameters of GaAs, InAs and AlAs were performed using ATK-force field code with Tersoff potentials (Nordlund et al. 2000, Hammerschmidt et al. 2008, Fichthor et al. 2011) as implemented in QuantumATK under the framework of Virtual Nano Lab. In this method, QuantumATK employs the Lagrangian strain and stress tensors. The strain and stress tensors are defined by $3 \mathrm{X} 3$ matrices using the Voigt notation, and they can be written compactly as 6vectors:

$$
\begin{aligned}
& \sigma=\left(\sigma_{x x}, \sigma_{y y}, \sigma_{z z}, \sigma_{x y}, \sigma_{x z}, \sigma_{y z}\right) \\
& \varepsilon=\left(\varepsilon_{x x}, \varepsilon_{y y}, \varepsilon_{z z}, \varepsilon_{x y}, \varepsilon_{x z}, \varepsilon_{y z}\right)
\end{aligned}
$$

Usually, the linear response of the stress to a strain vector is given as 


$$
\sigma=C \cdot \varepsilon
$$

The number of independent elements in matrix $C$ can be reduced depending on the crystal symmetry. For example, triclinic, monoclinic, orthorhombic, tetragonal II, rhombohedral, and hexagonal crystals, respectively have 21, 13, 9, 7, 6 and 5 independent elastic constants. However, in cubic crystal system, only 3 elastic constants $\left(C_{11}, C_{12}\right.$ and $\left.C_{14}\right)$ are totally independent as given by equation 3 (Born 1940, Mouhat and Coudert 2014),

$$
\left(\begin{array}{l}
\sigma_{1} \\
\sigma_{2} \\
\sigma_{3} \\
\sigma_{4} \\
\sigma_{5} \\
\sigma_{6}
\end{array}\right)=\left(\begin{array}{cccccc}
C_{11} & C_{12} & C_{12} & 0 & 0 & 0 \\
C_{12} & C_{11} & C_{12} & 0 & 0 & 0 \\
C_{12} & C_{12} & C_{11} & 0 & 0 & 0 \\
0 & 0 & 0 & C_{44} & 0 & 0 \\
0 & 0 & 0 & 0 & C_{44} & 0 \\
0 & 0 & 0 & 0 & 0 & C_{44}
\end{array}\right)\left(\begin{array}{c}
\varepsilon_{1} \\
\varepsilon_{2} \\
\varepsilon_{3} \\
\varepsilon_{4} \\
\varepsilon_{5} \\
\varepsilon_{6}
\end{array}\right)
$$

where $\sigma_{i}, \varepsilon_{i}$ and $C_{\mathrm{ijj}}$ are stress, strain and stiffness constants, respectively. To calculate the stiffness constants $C_{\mathrm{ij}}$, the atomic positions of each strained cell is first optimized. Then, QuantumATK (Atomistix ToolKit 2017.2) uses the universal linearly independent coupling strain (ULICS) vectors to minimize the number of stress calculations. For each strain vector three deformations $(-\eta, 0,+\eta)$ centered at $\eta=0$, is applied to simulate the cell along selected strains and calculate the corresponding stress vectors. Here, we use $\eta=0.002$, and number of intermediate deformations $n_{\eta}=3$ to filter out possible non-linear contributions. The highest polynomial order was taken as one in the stress against $\eta$ fitting. Contributions from the linear stress are obtained by fitting the stress $\sigma_{i}(\eta)$ curves of each Voigt stress and for every strain component. Then, taking crystal symmetry into account, the independent stiffness constants are calculated as the least-squares solution to a linear system of equations. The calculations were performed within a range of pressures, 0 - $100 \mathrm{GPa}$. Also, the code calculates the Poisson ratio, shear, bulk, and Young's modulus.

\section{Results and Discussion}

Elastic properties provide information about the dynamical and mechanical behavior of crystals and the nature of forces acting on the crystal. Mathematically, they are proportionality constants between stress and strain, which provides information on 
the interatomic stability of the crystal (Pokluda et al. 2015). As a result, any change on the stress or strain within the crystal has direct effect on the elastic constants (Cij). From the Born mechanical stability conditions for cubic structure, the elastic constants must satisfy the following necessary and sufficient conditions (Born 1940, Mouhat and Coudert 2014):

$$
\begin{aligned}
& C_{11}-C_{12}>0 ; \quad C_{44}>0 ; \quad C_{11}+2 C_{12}>0, \\
& C_{12}<B<C_{11}
\end{aligned}
$$

The calculated values of $C_{\mathrm{ij}}$ for GaAs are listed in Table 1 along with experimental and theoretical results available in the literature (Blakemore 1982, Varshney et al. 2010, Guler and Guler 2014). Table 1 shows that the results of $C_{\mathrm{ij}}$ for GaAs agree with other theoretical results (Varshney et al. 2010) at $0 \mathrm{GPa}$. The calculated results for $C_{11}, C_{12}$ and $C_{14}$ satisfy the structural and cubic stability conditions in Eq. (4) for gallium arsenide, indicating that cubic GaAs is mechanically stable within the highpressure regime $(0-100 \mathrm{GPa})$.

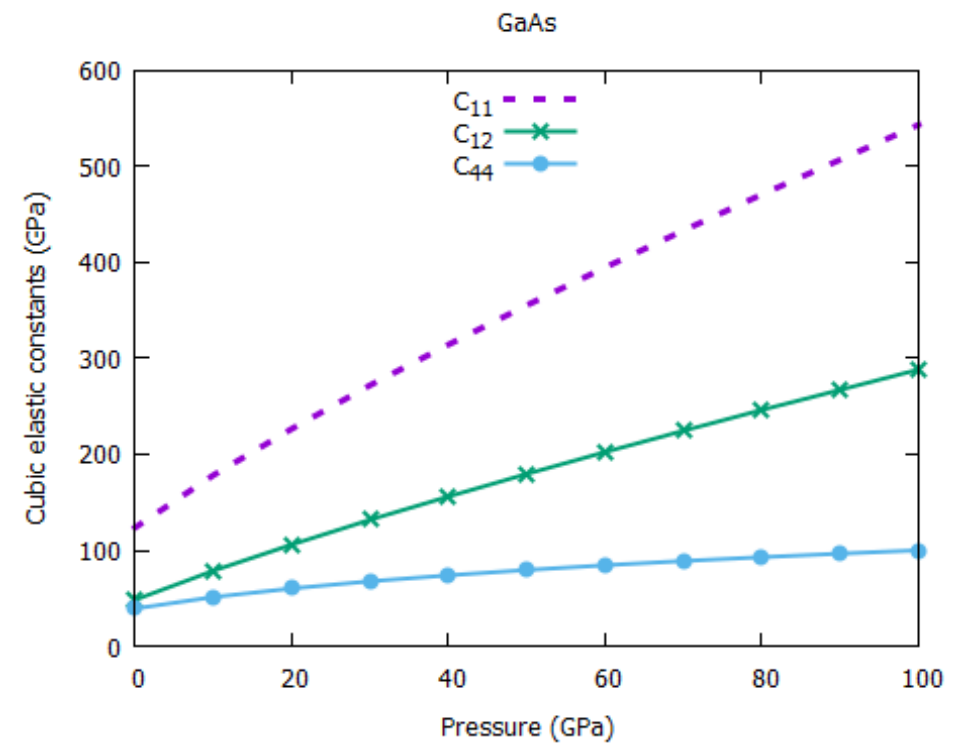

Fig. 1. The cubic elastic constants $C_{11}, C_{12}$ and $C_{14}$ for GaAs under pressure.

Figure 1 shows the stiffness constants at different pressures for GaAs. From these plots, all the calculated cubic stiffness constants are positive and increase monotonically as pressure increases. In addition, increase in $C_{11}$ is higher than $C_{12}$ and $C_{14}$. It is well known that $C_{11}$ represents the longitudinal stiffness behavior, $C_{12}$ represents the off-diagonal stiffness characteristics and $C_{44}$ explains the shear 
stiffness behavior of cubic crystals. As a result, longitudinal strain generates a change in volume without a corresponding change in shape. Therefore, this gives rise to larger change in $C_{11}$ since volume is related to pressure. A transverse strain (or shearing) causes a change in shape without a corresponding change in volume. Therefore, $C_{12}$ and $C_{14}$ are less sensitive to pressure compared to $C_{11}$ (Guler and Guler 2014).

The bulk modulus (B), shear modulus (G), Young's modulus (E), Pugh ratio (B/G), Poisson ratio $(v)$, Kleinman parameter $(\zeta)$ and Anisotropy factor A for GaAs at different pressures are listed in Table 1. The Young's modulus is defined as the ratio of stress to strain. It is the resistance to uniaxial tensions and is used to provide a measure of the stiffness of solids. This implies that, the higher the value of $\mathrm{E}$, the stiffer the material. This trend agrees with previous studies (Bing et al. 2010, Feng et al. 2014, Wang et al. 2014).

Table 1: Calculated elastic constants $C_{11}, C_{12}, C_{14}(\mathrm{GPa})$ and other related elastic parameters for GaAs at various pressure P (GPa). (B: bulk modulus (GPa), $G$ : shear modulus (GPa), $E$ : Young modulus $(\mathrm{GPa}), B / G$ : Pugh ratio, $v$ : Poisson ratio, $\zeta$ : Kleinman parameter, $A$ : Anisotropy factor.

\begin{tabular}{rllllllllll}
\hline $\mathrm{P}$ & $C_{11}$ & $C_{12}$ & $C_{14}$ & $B$ & $G$ & $E$ & $B / G$ & $v$ & $\zeta$ & $A$ \\
\hline 0 & 123.61 & 48.27 & 39.14 & 73.38 & 38.54 & 96.49 & 1.90 & 0.281 & 0.530 & 1.039 \\
& $\left(106.5^{\mathrm{a}}\right)$ & $\left(53.3^{\mathrm{a}}\right)$ & $\left(60.2^{\mathrm{a}}\right)$ & $\left(75.5^{\mathrm{a}}\right)$ & $\left(32.6^{\mathrm{a}}\right)$ & $\left(85.5^{\mathrm{a}}\right)$ & & $\left(0.31^{\mathrm{a}}\right)$ & \\
& $\left(106.5^{\mathrm{b}}\right)$ & $\left(60.2^{\mathrm{b}}\right)$ & $\left(33.6^{\mathrm{b}}\right)$ & $\left(75.6^{\mathrm{b}}\right)$ & $\left(28.9^{\mathrm{b}}\right)$ & $\left(63.0^{\mathrm{b}}\right)$ & & $\left(0.36^{\mathrm{b}}\right)$ & \\
& $(122.3-$ & $(40.6-$ & $(42.4-$ & $(70.8-$ & $\left(24.5^{\mathrm{c}}\right)$ & & & & & \\
& $\left.147.6^{\mathrm{c}}\right)$ & $\left.119^{\mathrm{c}}\right)$ & $\left.107^{\mathrm{c}}\right)$ & $\left.135^{\mathrm{c}}\right)$ & & & & & \\
10 & 178.29 & 78.62 & 51.13 & 111.84 & 50.60 & 130.16 & 2.21 & 0.306 & 0.574 & 1.026 \\
20 & 226.52 & 105.84 & 60.09 & 146.06 & 60.18 & 159.10 & 2.42 & 0.319 & 0.597 & 0.995 \\
30 & 272.02 & 131.76 & 67.55 & 178.51 & 68.57 & 186.03 & 2.60 & 0.326 & 0.611 & 0.963 \\
40 & 314.29 & 155.97 & 73.80 & 208.74 & 75.90 & 210.83 & 2.75 & 0.332 & 0.621 & 0.932 \\
50 & 355.30 & 179.54 & 79.34 & 238.12 & 82.65 & 234.76 & 2.88 & 0.336 & 0.629 & 0.902 \\
60 & 394.86 & 202.33 & 84.26 & 266.50 & 88.68 & 257.75 & 3.00 & 0.339 & 0.635 & 0.875 \\
70 & 433.37 & 224.57 & 88.71 & 294.16 & 94.68 & 280.07 & 3.10 & 0.341 & 0.640 & 0.849 \\
80 & 470.86 & 246.23 & 92.74 & 321.11 & 100.12 & 301.76 & 3.20 & 0.343 & 0.644 & 0.825 \\
90 & 507.08 & 267.18 & 96.38 & 347.14 & 105.20 & 322.68 & 3.29 & 0.345 & 0.647 & 0.803 \\
100 & 543.52 & 288.27 & 99.82 & 373.35 & 110.14 & 343.71 & 3.38 & 0.347 & 0.650 & 0.782 \\
\hline
\end{tabular}

${ }^{\mathrm{a}}$ Blakemore (1982), ${ }^{\mathrm{b}}$ Guler and Guler (2014), ${ }^{\mathrm{c}}$ Varshney et al. (2010)

It is clear from Table 1 that, pressure has significant effect on E. Thus, Young's modulus of GaAs shows a monotonic increase with pressure as displayed in Figure 2. 
This means that GaAs is stiffer at higher pressures because the higher the value of Young's modulus, the harder the material.

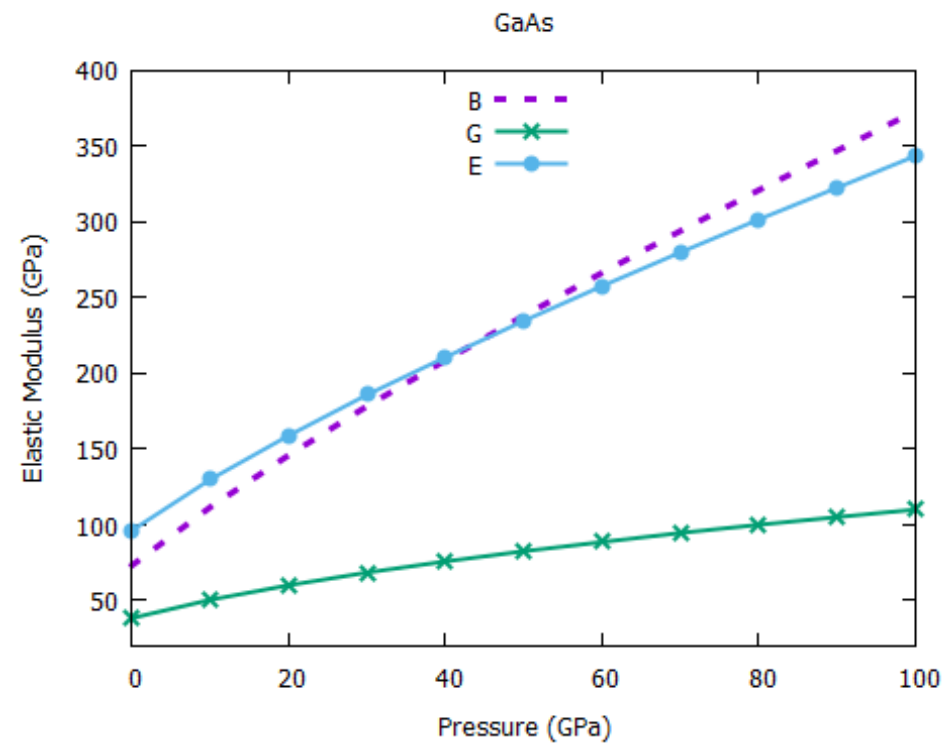

Fig. 2. Young, bulk, and shear modulus for GaAs under pressure.

Bulk modulus is defined as a measure of resistance to external deformation, and it gives a lot of information about bonding strength of materials (Bensalem et al. 2014, Guemou et al. 2014). Since pressure is directly proportional to bulk modulus, it is predictable that increase in pressure leads to corresponding increase in bulk modulus $(\Delta P=\Delta V \times B)$. Results obtained for $B$ as captured in Table 1 increase rapidly with pressure. As the pressure is increased from 0 to $100 \mathrm{GPa}$, the bulk modulus was observed to increase from 73.38 to $373.35 \mathrm{GPa}$ as displayed in Figure 2. The value obtained at zero pressure is consistent with experimental and theoretical results (Blakemore 1982, Guler and Guler 2014). In addition, shear modulus is known to measure the resistance to change in shape created by a shearing force. Results obtained for $G$ increased from 38.54 to $110.14 \mathrm{GPa}$ as the pressure is increased from 0 to $100 \mathrm{GPa}$. This result at zero pressure is consistent with experimental result (Blakemore 1982). This means that increase in pressure improves GaAs resistance to change in shape due to shearing forces.

Mechanical properties of solid materials can be investigated by employing the empirical Pugh ratio $(B / G)$ to determine if a material exhibits ductile or brittle behavior (Pugh 1954, Rahmati et al. 2014); i.e., if $B / G>1.75$, the material behaves in a ductile manner, whereas, if $B / G<1.75$ the material behaves in a brittle manner. 
Figure 3(a) is a plot of Pugh ratio against pressure up to $100 \mathrm{GPa}$. Pugh ratio analysis shows the ductile nature of gallium arsenide under pressure. From our calculations, the value of Pugh ratio is higher than 1.75 and increases with pressure. Therefore, GaAs can be classified as a ductile material under pressure up to $100 \mathrm{GPa}$, and pressure improves the ductility of this material.

Poisson ratio is defined as the ratio between the transverse strain and longitudinal strain in the elastic loading direction. It is utilized to reveal the stability of materials against shear and provides information about the type of bonding forces (Cao et al. 2013, Greaves et al. 2013). The larger values of Poisson's ratio favours plasticity of a material. For covalent materials $v=0.1$, ionic materials $v=0.25$, and values between 0.25 to 0.5 signifies that a central force exist in the solid material. In this study, the Poisson's ratio begins with 0.281 at zero pressure and increased to 0.347 with applied pressure as displayed in Figure 3(b). This signifies that those central forces are predominant in GaAs material. This result is consistent with previous studies.

(a) GaAs

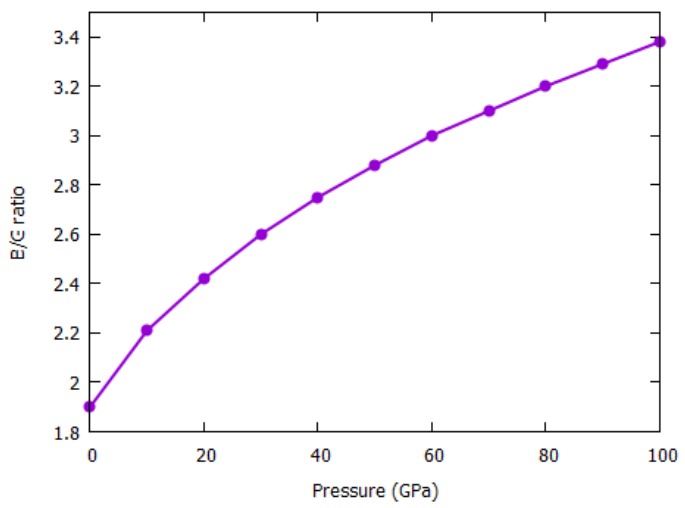

(b) GaAs

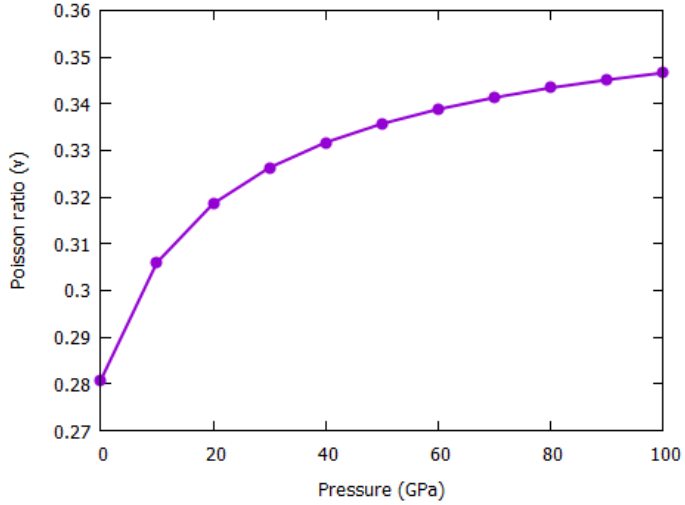

Fig. 3. (a) Pugh ratio $(B / G)$ and (b) Poisson's ratio for GaAs under pressure.

Kleinman parameter $(\zeta)$ describes the relative positions of anion and cation sublattices under volume conserving strain distortions for which positions are not fixed by symmetry. According to Harrison (1989), it is given by the relation:

$$
\zeta=\frac{C_{11}+8 C_{12}}{7 C_{11}+2 C_{12}}
$$

In a system, reducing bond stretching leads to $\zeta=1$, while reducing bond bending leads to $\zeta=0$. In this study, as the pressure increases, Kleinman parameter was observed to vary from 0.53 to 0.56 , indicating a decrease in bond stretching for 
GaAs. $\zeta$ grows monotonically with increase in pressure as shown in Figure 4(a), and increasing pressure reduces bond stretching.

(a)

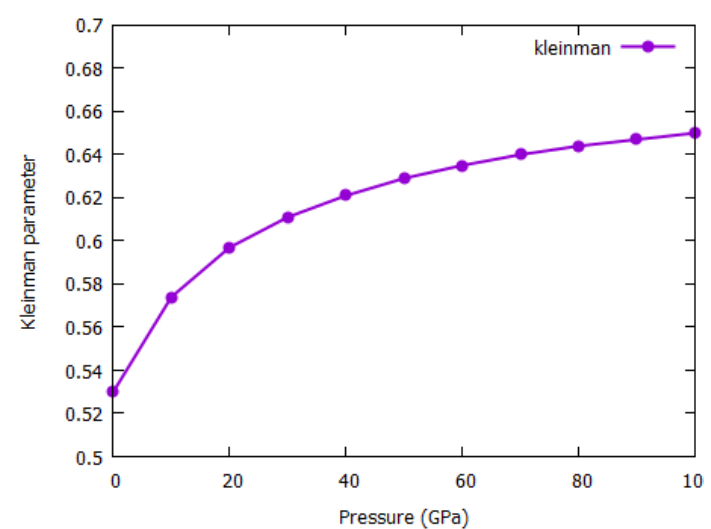

(b)

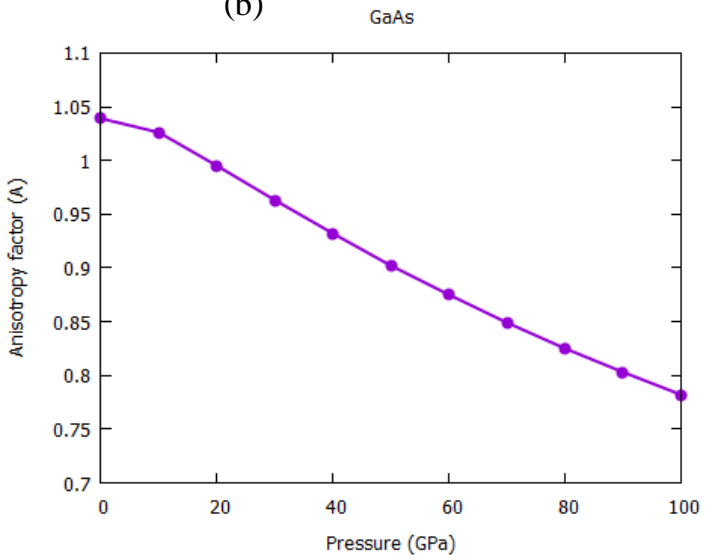

Fig. 4. (a) Kleinman parameter and (b) Anisotropy factor for GaAs under pressure.

Elastic anisotropy factor $(A)$ gives vital information about the degree of anisotropy in solid materials (Zener 1948). It has potential to influence micro-cracks in materials; as such it has vital implications in material engineering. $A=1$, implies the material under study is elastically isotropic and deform uniformly along all directions. Whereas, if $A>1$, the material is stiffest along the $<111>$ direction, and for $A<1$, it is stiffest along $\langle 100\rangle$ direction (Kabita et al. 2015). The value of $A$ is calculated at various pressures using the following relation (Maachou et al. 2011):

$$
A=\frac{2 C_{44}}{C_{11}-C_{12}}
$$

Figure 4(b) displays calculated values of $A$ against applied pressure. It was observed that the anisotropy factors are not equal to one, rather decreased from 1.039 to 0.782 with increasing pressure. This implies that, between $0-10 \mathrm{GPa}(A>1) \mathrm{GaAs}$ tends to be stiffest along $<111>$ body diagonals, and between $20-100 \mathrm{GPa}(A<1)$ gallium arsenide tends to be stiffest along $\langle 100\rangle$ cube axes.

The calculated elastic parameters for cubic indium arsenide under the effect of pressure up to $100 \mathrm{GPa}$ have been listed in Table 2. The mechanical stability conditions in Eq. (4) can validate the calculated stiffness constants within the entire pressure domain, 0 - $100 \mathrm{GPa}$. The obtained results of elastic constants for InAs meet the stability conditions, indicating that they are mechanically stable at high-pressure. 
The calculated results for elastic constants at $0 \mathrm{GPa}$ are slightly higher than both experimental and theoretical values. This might be associated with the potential force field used in this calculation. However, this does not affect the validity of these results, since they are within the acceptable limits of calculations using different classical potential force field.

Table 2: Calculated elastic constants $C_{11}, C_{12}, C_{14}(\mathrm{GPa})$ and other related elastic parameters for InAs at various pressure $P(\mathrm{GPa})$. ( $B$ : bulk modulus $(\mathrm{GPa}), G$ : shear modulus $(\mathrm{GPa}), E$ : Young modulus $(\mathrm{GPa}), B / G$ : Pugh ratio, v: Poisson ratio, $\zeta$ : Kleinman parameter, $A$ : Anisotropy factor, $A^{*}$ : degree of elastic anisotropy).

\begin{tabular}{rlcccccccccc}
\hline $\mathrm{P}$ & $C_{11}$ & \multicolumn{1}{c}{$C_{12}$} & \multicolumn{1}{c}{$C_{44}$} & $B$ & $G$ & $E$ & $B / G$ & $v$ & $\zeta$ & $A$ & $A^{*}\left(10^{-2}\right)$ \\
\hline 0 & 113.58 & 55.92 & 54.69 & 75.13 & 42.29 & 76.67 & 1.78 & 0.3299 & 0.6185 & 1.897 & 4.84 \\
& $\left(83.29^{\mathrm{b}}\right)$ & $\left(45.26^{\mathrm{b}}\right)$ & $\left(39.59^{\mathrm{b}}\right)$ & $\left(58^{\mathrm{e}}\right)$ & & & & & & & \\
& $\left(83.0^{\mathrm{a}}\right)$ & $\left(46.7^{\mathrm{a}}\right)$ & $\left(37.2^{\mathrm{c}}\right)$ & $\left(71.85^{\mathrm{c}}\right)$ & & & & & & & \\
& $\left(84.1^{\mathrm{c}}\right)$ & $\left(48.1^{\mathrm{c}}\right)$ & & & & & & & & & \\
10 & 161.65 & 88.06 & 67.71 & 112.58 & 53.01 & 99.53 & 2.12 & 0.3527 & 0.6624 & 1.840 & 4.39 \\
20 & 204.69 & 117.39 & 77.02 & 146.49 & 61.32 & 119.11 & 2.39 & 0.3645 & 0.6858 & 1.764 & 3.81 \\
30 & 244.38 & 144.76 & 84.14 & 177.96 & 68.18 & 136.67 & 2.61 & 0.3720 & 0.7011 & 1.689 & 3.26 \\
40 & 282.33 & 171.14 & 89.90 & 208.20 & 74.14 & 153.14 & 2.81 & 0.3774 & 0.7122 & 1.617 & 2.74 \\
50 & 318.45 & 196.40 & 94.58 & 237.08 & 79.33 & 168.59 & 2.99 & 0.3815 & 0.7207 & 1.549 & 2.28 \\
60 & 252.94 & 220.65 & 98.40 & 264.74 & 83.91 & 183.18 & 3.16 & 0.3847 & 0.7274 & 1.487 & 1.87 \\
70 & 387.23 & 244.84 & 101.64 & 292.30 & 88.12 & 197.55 & 3.32 & 0.3874 & 0.7330 & 1.427 & 1.51 \\
80 & 420.51 & 268.39 & 104.31 & 319.09 & 91.91 & 211.37 & 3.47 & 0.3896 & 0.7377 & 1.371 & 1.18 \\
90 & 452.90 & 291.38 & 106.49 & 345.22 & 95.32 & 224.75 & 3.62 & 0.3915 & 0.7417 & 1.318 & 0.99 \\
100 & 484.23 & 313.66 & 108.23 & 370.51 & 98.38 & 237.61 & 3.77 & 0.3931 & 0.7452 & 1.269 & 0.68 \\
\hline
\end{tabular}

${ }^{\mathrm{a}}$ Ellaway and Faux (2003), ${ }^{\mathrm{b}}$ Gerlich (1963), ${ }^{\mathrm{c}}$ Victor et al. $(2017),{ }^{\mathrm{d}}$ Hellwege et al. (1982)

Due to difficulty in measuring high pressure dependence of elastic parameters, there are no experimental values for InAs in the literature to compare with. Therefore, these results can be used as the basis for experimental work at high pressures for indium arsenide material. It is clear from Table 2 that, $C_{11}, C_{12}$ and $C_{44}$ increase with increasing pressure. Also, $C_{11}$ is more sensitive to change in pressure compared to $\mathrm{C}_{12}$ and $C_{44}$. Results obtained for bulk modulus $B$ at zero pressure is consistent with other theoretical studies (Victor et al. 2017). As the pressure is increased to $100 \mathrm{GPa}, B$ was observed to increase rapidly to $370.51 \mathrm{GPa}$. This means that increase in pressure enhances the resistance to external deformation for InAs. Shear modulus $G$ for InAs was observed to increase monotonically with pressure. This indicates that the higher the pressure the larger the value of $G$. Hence, InAS becomes more resistant to change in shape due to shearing forces at high pressure. In Table 2, one can observe that 
Young's modulus $E$ is sensitive to pressure. $E$ increased from 76.67 to $237.61 \mathrm{GPa}$ as applied pressure is increased from 0 to $100 \mathrm{GPa}$, meaning an increase in induced pressure improves the stiffness of indium arsenide, since Young's modulus is a measure of stiffness of solid materials. This implies that InAs becomes extremely hard to be broken at high-pressure. Pugh ratio $B / G$ was observed to increase from 1.78 to 3.77 within the pressure range. Since $B / G>1.75$, it means that indium arsenide behaves as a ductile material from low to high pressure regime, and the ductility of this material increases with pressure.

Poisson ratio $v$ for this material was observed to increase from 0.3299 to 0.3931 as induced pressure increases from 0 to $100 \mathrm{GPa}$. This indicates that central force exists in this material since $v>0.25$, and Poisson ratio increases with pressure. This is consistent with Victor et al. (2017). The Kleinman parameter which determines bond bending and bond stretching was observed to be 0.6185 at zero pressure and 0.7452 at $100 \mathrm{GPa}$. This means that pressure reduces bond stretching in InAs, similar to our observation in gallium arsenide material. Furthermore, it was observed that elastic anisotropy factor $A$ is not equal to one, indicating the existence of elastic anisotropy in InAs. From Table 2, increase in pressure reduces $A$ from 1.897 to 1.269 for InAs. This means that InAs is stiffest along <111> body diagonals. For cubic crystals, the degree of elastic anisotropy $\left(A^{*}\right)$ is calculated as $A^{*}=3(A-1)^{2} /\left(3(A-1)^{2}+25 A\right)$ (Bing et al. 2010). In this study, $A^{*}$ was found to decrease from 0.0484 to 0.0068 as induced pressure is increased from 0 to $100 \mathrm{GPa}$.

Table 3. Calculated elastic constants $C_{11}, C_{12}, C_{14}$ and other related elastic parameters for AlAs at various pressure $P$. (B: bulk modulus, $G$ : shear modulus, $E$ : Young modulus, $B / G$ : Pugh ratio, $v$ : Poisson ratio).

\begin{tabular}{cccccrrrr}
\hline $\begin{array}{l}\mathrm{P} \\
(\mathrm{GPa})\end{array}$ & $\begin{array}{c}C_{11} \\
(\mathrm{GPa})\end{array}$ & \multicolumn{1}{c}{$\begin{array}{c}C_{12} \\
(\mathrm{GPa})\end{array}$} & $\begin{array}{c}C_{44} \\
(\mathrm{GPa})\end{array}$ & $\begin{array}{c}B \\
(\mathrm{GPa})\end{array}$ & $\begin{array}{c}G \\
(\mathrm{GPa})\end{array}$ & $\begin{array}{c}E \\
(\mathrm{GPa})\end{array}$ & $B / G$ & $v$ \\
\hline 0 & 120.77 & 57.34 & 67.26 & 78.48 & 49.73 & 83.84 & 1.58 & 0.3220 \\
& $\left(120^{\mathrm{a}}\right)$ & $\left(58.2^{\mathrm{a}}\right)$ & $\left(57.6^{\mathrm{a}}\right)$ & & & & & $\left(0.328^{\mathrm{a}}\right)$ \\
& $\left(119^{\mathrm{b}}\right)$ & $\left(54.8^{\mathrm{b}}\right)$ & $\left(60.4^{\mathrm{b}}\right)$ & & & & & \\
& $\left(116^{\mathrm{c}}\right)$ & $\left(55^{\mathrm{c}}\right)$ & $\left(57^{\mathrm{c}}\right)$ & & & & & \\
10 & 170.37 & 90.81 & 83.27 & 117.33 & 61.90 & 107.21 & 1.89 & 0.3477 \\
20 & 214.83 & 121.54 & 94.70 & 152.54 & 71.36 & 126.99 & 2.14 & 0.3613 \\
30 & 255.92 & 150.37 & 103.95 & 185.55 & 79.18 & 144.60 & 2.34 & 0.3701 \\
40 & 294.92 & 178.04 & 111.33 & 217.00 & 85.95 & 160.88 & 2.52 & 0.3764 \\
50 & 64987.01 & 12078.70 & 31321.77 & 29714 & 29274.61 & 61200.76 & 1.01 & 0.1567 \\
60 & 67358.46 & 7352.81 & 31996.50 & 27354.69 & 31183.54 & 65911.18 & 0.877 & 0.0948 \\
70 & 67492.72 & 7067.77 & 32036.41 & 27209.41 & 31293.99 & 66152 & 0.869 & 0.0948 \\
80 & 67625.79 & 6783.16 & 32076.41 & 27064.03 & 31403.79 & 66389 & 0.818 & 0.0912 \\
90 & 67757.73 & 6498.87 & 32116.18 & 26918.49 & 31512.98 & 66620 & 0.854 & 0.0875 \\
100 & 288.15 & 173.22 & 110.13 & 211.52 & 84.82 & 158.08 & 2.490 & 0.3754 \\
\hline
\end{tabular}

${ }^{\mathrm{a}}$ Gehrsitz et al. (1999), ${ }^{\mathrm{b}} \mathrm{Li}$ et al. (2013), ${ }^{\mathrm{c}}$ Chetty et al. (1989) 
For AlAs, results obtained at zero pressure are in agreement with both experimental and theoretical values (Chetty et al. 1989, Gehrsitz et al. 1999, Li et al. 2013). All calculated elastic parameters increased with pressure up to $40 \mathrm{GPa}$ (Table $3)$. Beyond this point, we observed a drastic jump in the values of $C_{11}, C_{12}, C_{44}, B, G$ and $E$, and sudden drop in values of $v$ and $B / G$. This behavior was observed between 50 to $90 \mathrm{GPa}$. Again, at $100 \mathrm{GPa}$ there was sudden drop in the values of $C_{11}, C_{12}$, $C_{44}, B, G$ and $E$. Whereas, a jump in values of $v$ and $B / G$ was observed. This behavior might be associated with phase transition that might have occurred at these respective pressures. It was observed experimentally that between $12.4-14.2 \mathrm{GPa}$, zincblende AlAs undergoes phase transition to nickel arsenide phase (NiAl-AlAs) (Foyen and Cohen 1983, Weinstein et al. 1987). Theoretically, it has been reported that at 77.9 GPa phase transition from NiAl-AlAs to CsCl-AlAs phase was observed (Mujica et al. 1995).

The calculated values of stiffness constants $\left(C_{11}, C_{12}, C_{44}\right)$ and $B$ modulus support both the structural and cubic stability conditions for AlAs within the pressure range 50 - $90 \mathrm{GPa}$. However, the cubic stability condition $C_{12}<B<C_{11}$ was violated since $C_{12}>B$ at $100 \mathrm{GPa}$. This might be as a result of phase transition to the unstable $\mathrm{CsCl}$ AlAs phase at $100 \mathrm{GPa}$. This implies that AlAs becomes unstable at high pressure above $100 \mathrm{GPa}$. From our results, at zero pressure, 50, 60, 70, 80 and $90 \mathrm{GPa}$, Pugh ratio is less than 1.75. This suggests that AlAs is brittle at these pressures. Whereas, from 10 - $40 \mathrm{GPa}$ and $100 \mathrm{GPa}$ AlAs material shows ductile behavior. Additionally, Poisson's ratio for this material was observed to decrease as pressure increase from 50 to $90 \mathrm{GPa}$. Since Poisson's ratio suggests stability of crystal against shear deformation, larger values implies better plasticity. Therefore, between the pressures 0 - $40 \mathrm{GPa}$, AlAs show better plasticity behavior, and between $50-90 \mathrm{GPa}$, AlAs exhibits lesser plasticity.

\section{Conclusions}

This work has described the elastic and mechanical properties of GaAs, InAs and AlAs under high pressure up to $100 \mathrm{GPa}$. Results obtained at zero pressure are consistent with some of the experimental and theoretical values previously reported. According to the Born mechanical stability condition, GaAs and InAs are mechanically stable under the entire high-pressure region, while for AlAs the stability condition is violated at $100 \mathrm{GPa}$. Calculated Young, bulk and shear moduli, Poisson ratio, anisotropy factor, degree of anisotropy, Kleinman parameter and Pugh ratio indicated that the ductility of GaAs and InAs will be enhanced with pressure. The aluminum arsenide material was found to be brittle under 50 - $90 \mathrm{GPa}$ and unstable at $100 \mathrm{GPa}$. 


\section{Acknowledgements}

The authors acknowledge the Federal University of Petroleum Resources Effurun for providing the Mini Workstation used for the calculations and QuantumATK for access to the VNL-ATK software package. Also, we wish to acknowledge the two anonymous reviewers for their meaningful comments to make this manuscript better.

\section{References}

Atomistix ToolKit 2017.2. Quantumwise A/S, www.quantumwise.com

Bensalem S, Chegaar M, Maouche D, Bouhemadou A. 2014. Theoretical study of structural, elastic and thermodynamic properties of CZTX $(\mathrm{X}=\mathrm{S}$ and $\mathrm{Se})$ alloys. Journal of Alloys and Compounds 589: 137-142. doi.org/10.1016/j.jallcom.2013.11.113.

Bing L, Rong-Feng L, Yong Y, Xiang-Dong Y. 2010. Characterisation of the high-pressure structural transition and elastic properties in boron arsenic. Chinese Physics $B \quad 19$ (7): 076201. doi:10.1088/1674-1056/19/7/076201.

Blakemore JS. 1982. Semiconducting and other major properties of gallium arsenide. Journal of Applied Physics 53 (10): R123-R181. doi:10.1063/1.331665

Born M. 1940. On the stability of crystal lattices. I. Mathematical Proceedings of the Cambridge Philosophical Society. 36: 160 - 172. doi:10.1017/S0305004100017138.

Born M, Huang K, Lax M. 1955. Dynamical Theory of Crystal Lattices. American Journal of Physics, 23 (7): 474-474. doi:10.1119/1.1934059.

Cao Y, Zhu J, Liu Y, Nong Z, Lai Z. 2013. First-principles studies of the structural, elastic, electronic and thermal properties of $\mathrm{Ni}_{3} \mathrm{Si}$. Computational Materials Science 69: 4045. doi:10.1016/j.commatsci.2012.11.037

Chetty N, Muoz A, Martin RM. 1989. First-principles calculation of the elastic constants of AlAs. Physical Review B 40 (17): 11934-11936. doi:10.1103/physrevb.40.11934.

Chun-Lei W, Ben-Hai Y, Hai-Liang H, Dong C, Hai-Bin S. 2009. First principles study on the elastic and thermodynamic properties of $\mathrm{TiB}_{2}$ crystal under high temperature. Chinese Physics $B 18$ (3): 1248.

Feng L, Li N, Yang M, Liu Z. 2014. Effect of pressure on elastic, mechanical and electronic properties of WSe 2 A first-principles study. Materials Research Bulletin, 50: 503508. doi:10.1016/j.materresbull.2013.11.016.

Gehrsitz S, Sigg H, Herres N, Bachem K, Köhler K, Reinhart FK. 1999. Compositional dependence of the elastic constants and the lattice parameter of AlxGa ${ }_{1-x}$ As. Physical Review B 60 (16): 1160111610. doi:10.1103/physrevb.60.11601.

Guemou M, Abdiche A, Riane R, Khenata R. 2014. Ab initio study of the structural, electronic, and optical properties of BAs and $\mathrm{BN}$ compounds and $\mathrm{BN}_{\mathrm{x}} \mathrm{As}_{1-\mathrm{x}}$ alloys. Physica B: Condensed Matter 436: 33-40. doi:10.1016/j.physb.2013.11.030.

Güler E, Güler M. 2015. Elastic and mechanical properties of hexagonal diamond under pressure. Applied Physics A 11 (2): 721-726. doi:10.1007/s00339-015-9020-8.

Guler M, Guler E. 2014. High pressure phase transition and elastic behavior of europium oxide. Journal of Optoelectronic and Advanced Materials 16 (11-12): 1322-1327.

Güler E, Güler M. 2014. Phase transition and elasticity of gallium arsenide under pressure. Materials Research 17 (5): 1268-1272. doi:10.1590/1516-1439.272414.

Greaves GN, Greer AL, Lakes RS, Rouxel T. 2011. Poisson's ratio and modern materials. Nature Materials 10 (11): 823-837. doi:10.1038/nmat3134.

Harrison AW. 1989. Electronic Structure and Properties of Solids, New York: Dover. 
Hong-Lin C, Xiang-Rong C, Guang-Fu J, Dong-Qing W. 2008. Structures and Phase Transition of GaAs under Pressure. Chinese Physics Letters 25 (6): 2169-2172. doi:10.1088/0256-307x/25/6/067.

Jun Z, Jing-Xin Y, Yan-Ju W, Xiang-Rong C, Fu-Qian J. 2008. First-principles calculations for elastic properties of rutile $\mathrm{TiO} 2$ under pressure. Chinese Physics B 17(6): 2216-2221.

Kabita K, Jameson M, Sharma BI, Brojen RK, Thapa RK. 2016. A detailed first principles study on the structural, elastic, and electronic properties of indium arsenide (InAs) under induced pressure. Canadian Journal of Physics 94 (3): 254-261. doi:10.1139/cjp-2015-0275.

Li X-X, Tao X-M, Chen H-M, Ouyang Y-F, Du Y. 2013. The pressure dependences of elastic and lattice dynamic properties of AlAs from ab initio calculations. Chinese Physics $B$ 22 (2): 026201. doi:10.1088/1674-1056/22/2/026201.

Liu CG, Lu WZ, Klein MB. 1995. Pressure-induced phase transformations in AlAs: Comparison between ab initio theory and experiment. Physical Review B 51: 5678. doi: 10.1103/PhysRevB.51.5678.

Louail L, Maouche D, Hachemi A. 2006. Elastic properties of InAs under pressure up to $18 \mathrm{GPa}$. Materials Letters 60 (27): 3269-3271. doi:10.1016/j.matlet.2006.03.011.

Mouhat F, Coudert F-X. 2014. Necessary and sufficient elastic stability conditions in various crystal systems. Physical Review B 90 (22): 4104. doi:10.1103/physrevb.90.224104.

Na-Na L, Ren-Bo S, Da-Wei D. 2009. Elastic constants and thermodynamic properties of Mg2SixSn1-x from first-principles calculations. Chinese Physics B 18 (5): 1979.

Pokluda J, Černý M, Šob M, Umeno Y. 2015. Ab initio calculations of mechanical properties: Methods and applications. Progress in Materials Science, 73: 127-158. doi: 10.1016/j.pmatsci.2015.04.001.

Pugh SF. 1954. Relation between the elastic moduli and the plastic properties of polycrystalline pure metals. Philosophical Magazine and Journal of Science 45 (367): 823843. doi:10.1080/14786440808520496.

Rahmati A, Ghoohestani M, Badehian H, Baizaee M. 2014. Ab. initio study of the structural, elastic, electronic and optical properties of Cu3N. Materials Research 17 (2): 303-310. doi: 10.1590/s1516-14392014005000039.

Varshney D, Joshi G, Varshney M, Shriya S. 2010. Pressure dependent elastic and structural (B3-B1) properties of $\mathrm{Ga}$ based monopnictides. Journal of Alloys and Compounds 495 (1): 2332. doi:10.1016/j.jallcom.2010.01.077.

Venkateswaran UD, Cui LJ, Weinstein BA, Chambers FA. 1992. Forward and reverse high-pressure transitions in bulklike AlAs and GaAs epilayers. Physical Review B 45(16): 92379247. doi:10.1103/physrevb.45.9237.

Wang S, Li, J-X, Du Y-L, Cui C. 2014. First-principles study on structural, electronic and elastic properties of graphene-like hexagonal $\mathrm{Ti}_{2} \mathrm{C}$ monolayer. Computational Materials Science, 83: 290293. doi:10.1016/j.commatsci.2013.11.025.

Zi-Jiang L, Jian-Hong Q, Yuan G, Qi-Feng C, Ling-Cang C, Xiang-Dong Y. 2007. Thermoelasticity of $\mathrm{CaO}$ from first principles. Chinese Physics 16 (2): 499-505. doi: 10.1088/1009-1963/16/2/035. 